\title{
Analysis of Nosocomial Infection and Risk Factors in Patients with ECMO Treatment
}

\author{
Zhan-jie $\mathrm{Li}^{1}{ }^{1} *$ \\ Dong-Fang Zhang ${ }^{2, *}$ \\ Wei-Hong Zhang ${ }^{3}$ \\ 'Department of Infection Control, First \\ Affiliated Hospital of Nanjing Medical \\ University, Nanjing, 210029, Jiangsu, \\ People's Republic of China; ${ }^{2}$ Department \\ of Infection Control, Shanghai Fourth \\ People's Hospital Affiliated to Tongji \\ University School of Medicine, Shanghai, \\ 200434, People's Republic of China; \\ ${ }^{3}$ Office of the Dean, Jiangsu Shengze \\ Hospital Affiliated to Nanjing Medical \\ University, Suzhou, Jiangsu, 215228, \\ People's Republic of China
}

*These authors contributed equally to this work
Correspondence: Wei-Hong Zhang Office of the Dean, Jiangsu Shengze Hospital Affiliated to Nanjing Medical University, No. 1399, Shichang West Road, Shengze Town, Wujiang District, Suzhou, 215228, Jiangsu, People's Republic of China

Tel +86-5 I2-63097006

Email Weihong02092123@163.com
Objective: To investigate the drug resistance of nosocomial infection-related pathogens in patients who underwent extracorporeal membrane oxygenation (ECMO), analyzing the nosocomial infection-related risk factors.

Methods: The medical records of 56 patients who received ECMO support treatment in the First Affiliated Hospital with Nanjing Medical University from January 2013 to December 2019 were selected. The nosocomial infection, pathogen distribution and drug resistance, and the influencing factors of nosocomial infection were analyzed. The predictive value of independent risk factors for nosocomial infection after ECMO was analyzed using the receiver operating characteristic (ROC) curve.

Results: A total of 56 patients receiving ECMO treatment were included. The nosocomial infection rate was $28.57 \%$, and the prevalence infection rate was $44.64 \%$. Lower respiratory tract infection was the main infection site. Among these infectious patients, 53 strains of pathogens were detected. The results showed that the gram-negative bacteria were mainly Acinetobacter baumannii and Klebsiella pneumonia. Moreover, the drug resistance rate of Acinetobacter baumannii to most of the antibiotics was more than $65 \%$, among which the drug resistance rate to carbapenems was $80 \%$. The results of risk factors of nosocomial infection after ECMO were analyzed by univariate analysis, showing that ECMO treatment time, hospitalization time, antibacterial drug use time, ventilator use time, catheter intubation time and central venous intubation time were statistically significant (all $p<0.001$ ). Multivariate analysis identified that ECMO treatment time was an independent risk factor. As showed by ROC curve, ECMO treatment time had a high predictive value for postoperative nosocomial infection. ECMO treatment times of more than 4.5 days were associated with an increased risk of nosocomial infection.

Conclusion: The nosocomial infection rate after ECMO was relatively high, and the main pathogens are Gram-negative bacteria. The selection of antibiotics should be based on the results of pathogen drug sensitivity.

Keywords: extracorporeal membrane oxygenation, nosocomial infection, risk factors, infection site

\section{Introduction}

Extracorporeal membrane oxygenation (ECMO), as an extracorporeal life support technology, is widely applied in patients with severe cardiopulmonary failure, which could maintain cardiopulmonary respiration and cardiopulmonary bypass (CPB) and help gain time for primary disease treatment and cardiopulmonary function recovery. ${ }^{1,2}$ ECMO originated in the late 1960s, and it has been successfully applied in treating a patient with adult respiratory distress syndrome (ARDS) in $1971 .^{3}$ In 1989 , the in vitro life support 
organization was established in the United States, which has become an important international academic exchange platform for ECMO data collection and analysis, and the evaluation of patient's prognosis. ${ }^{4}$ The clinical application of ECMO in China began in the 1990s, and ECMO has been applied in more than 5000 cases in China in 2019. At present, there are about 500 ECMO, and 260 hospitals can provide ECMO services in China. ${ }^{5}$ With the continuous progress and application of ECMO technology, ECMO application has been gradually accepted by the public. ${ }^{6}$

Although ECMO is a CPB technology, the incidence of complications in ECMO support treatment is $75.8 \%{ }^{7}$ Bleeding, renal failure and secondary infection are common complications after ECMO. According to the "Infectious Disease Task Force", created in 2008 to control the infection of ECMO, about $20 \%$ of adults who receive ECMO are at risk of nosocomial infections. ${ }^{8}$ Nosocomial infection after ECMO, as one of its complications, dramatically increases the mortality of patients. ${ }^{9}$ Zhou et al $^{10}$ analyzed the distribution and drug resistance of pathogens causing nosocomial infections in patients treated with ECMO; however, no analyses on nosocomial infection-related risk factors have been made. Li et al ${ }^{11}$ indicated the significant correlations between the secondary infections of post-cardiac surgery extracorporeal membrane oxygenation supportive treatment (pCS-ECMO) and mechanical ventilation time, ICU residence, ECMO duration, and total hospital stay. However, there are no appropriate guidelines and prognostic factors for antimicrobial prophylaxis in patients with ECMO. ${ }^{12,13}$ To make the effective protocol of antibiotic application, it is crucial to figure out the profiling of nosocomial infections and risk factors.

In this study, 56 patients who received ECMO support treatment at the First Affiliated Hospital with Nanjing Medical University from January 2013 to December 2019 were investigated for nosocomial infection. The distribution of pathogens, drug resistance and the related risk factors were analyzed. The predictive value of nosocomial infection after ECMO was analyzed using the receiver operating characteristic (ROC) curve. This study aims to provide a reference for the prevention and reduction of nosocomial infection after ECMO.

\section{Research Objects and Methods Research Objects}

The medical records of 56 patients who received ECMO support treatment at the First Affiliated Hospital with Nanjing Medical University from January 2013 to
December 2019 were included. The nosocomial infection after ECMO surgery was defined as the nosocomial infection that occurred between $24 \mathrm{~h}$ after ECMO to $48 \mathrm{~h},{ }^{14}$ based on the Diagnostic Criteria for Nosocomial Infections (Proposed) issued by the Ministry of Health of the P.R. China (2001). The patients were divided into the infection group (nosocomial infection after ECMO; $n=16$ ) and the non-infection group (no nosocomial infection after ECMO; $\mathrm{n}=40$ ). The inclusive criteria were as follows: (1) age > 18 years old; (2) no evidence of infection (such as in clinical manifestations and specimen culture) before ECMO support treatment; (3) ECMO support treatment time $\geq 48 \mathrm{~h}$. The exclusion criteria included: (1) infection before ECMO support treatment; (2) weaning or death within $48 \mathrm{~h}$ after ECMO support treatment.

\section{Methods}

\section{Observation Indexes}

The retrospective study was performed. The condition (such as gender, age, hypertension, diabetes and nosocomial infection), ECMO treatment mode, installation mode, and time of ECMO treatment, hospitalization, antibacterial drug use, combination medication, ventilator use, central venous intubation and catheter intubation of patient in the infection group and the non-infection group were collected and recorded from the hospital information system.

\section{Bacterial Identification and Drug Sensitivity Test}

The samples were collected from patients with nosocomial infection after ECMO surgery, namely the specimens from infected sites, including bronchoalveolar fluid, sputum, blood, midstream urine, feces and pleural effusion. The isolated colonies were identified using the VITEK-2 Compact automatic bacteria identification instrument (Biomerieux, Marcy l'Etoile, France) or API system (Biomerieux). The quality control strains (National Center for Clinical Laboratories) were as follows: Escherichia coli (ATCC25922), Klebsiella pneumonia (ATCC700603), Staphylococcus aureus (ATCC25923), Acinetobacter baumannii (ATCC19606), Pseudomonas aeruginosa (ATCC278553) and Enterobacter cloacae (ATCC700323). Bacterial drug sensitivity test was performed using VITEK-2 Compact automatic bacteria identification instrument (Biomerieux) or the disk diffusion method. The drug sensitivity results were judged according to the standard of Clinical and Laboratory Standards Institute (2019). ${ }^{15}$ 


\section{Statistical Analysis}

SPSS 26.0 software was used for data sorting and analysis. The counting data were expressed as a constituent ratio $(\%)$, and analyzed by $\mathrm{X}^{2}$ test. The measurement data were shown as $(\bar{X} \pm S)$ or M (P25-P75). For measurement data in a normal distribution, the comparison between groups was analyzed by $t$-test. The comparison between groups was analyzed by the Mann-Whitney $U$-test to check the skewness distribution. The factors with statistically significant differences in infection after ECMO were further analyzed by univariate analysis, and the independent risk factors were analyzed by binary Logistic regression. The ROC curve was used to analyze the predictive value of independent risk factors for nosocomial infection after ECMO, and to predict the optimal critical value, sensitivity and specificity of nosocomial infection after ECMO. Area under ROC curve (AUC) closer to 1 indicates the better diagnostic effect. AUC in 0.5-0.7 suggests low accuracy, AUC in 0.7-0.9 suggests certain accuracy, and AUC in 0.9 above suggests a high accuracy. $p<0.05$ indicates the difference was statistically significant.

\section{Results}

\section{Nosocomial Infection After ECMO}

A total of 16 cases in 56 patients developed the nosocomial infection after ECMO, with an infection rate of $28.57 \%$. The prevalence infection rate was $44.64 \%$. Among 56 patients, there were 33 males and 23 females. The main site of nosocomial infection was located at lower respiratory tract infection (18 cases, $72.00 \%$ ), followed by blood infection (3 cases, $12.00 \%$ ). The distribution and constituent ratios of nosocomial infection sites are listed in Table 1.

\section{Distribution of Pathogens}

A total of 53 strains of pathogens were detected in patients with ECMO postoperative infection, which were mainly Gram-negative bacteria (45 strains, $84.91 \%$ ) and fungi (8 strains, $15.09 \%$ ). The majority of gram-negative bacteria were Acinetobacter baumannii (15 strains, 28.30\%) and Klebsiella pneumonia ( 8 strains, $15.09 \%$ ), and the majority of fungi were Candida albicans (3 strains, 5.66\%) and Candida glabrata (3 strains, 5.66\%). No Gram-positive bacteria were detected (Table 2).

\section{Drug Resistance of Main Pathogens}

Gram-negative bacteria mainly included Acinetobacter baumannii and Klebsiella pneumonia. The resistance rate of
Table I Distribution and Constituent Ratio of Nosocomial Infection Site

\begin{tabular}{|l|c|c|}
\hline Infection Site & $\begin{array}{c}\text { Infection Case } \\
\text { Numbers } \\
(\mathbf{n = 2 5 )}\end{array}$ & $\begin{array}{c}\text { Constituent } \\
\text { Ratio (\%) }\end{array}$ \\
\hline Lower respiratory tract & 18 & 72.00 \\
Blood & 3 & 12.00 \\
CAUTI & 1 & 4.00 \\
Surgical site & I & 4.00 \\
Antibiotic-associated diarrhea & I & 4.00 \\
Pleural cavity & I & 4.00 \\
\hline
\end{tabular}

Note: Antibiotic-associated diarrhea: diarrhea related to the intestinal dysbacteriosis, which caused by antibiotics.

Abbreviation: CAUTI, catheter-associated urinary tract infection.

Table 2 Distribution of Nosocomial Infection Pathogens Species

\begin{tabular}{|l|c|c|}
\hline Pathogens & $\begin{array}{c}\text { Strains } \\
\text { (n=53) }\end{array}$ & $\begin{array}{c}\text { Constituent } \\
\text { Ratio (\%) }\end{array}$ \\
\hline Gram-negative bacteria & 45 & $84.91 \%$ \\
Acinetobacter baumannii & 15 & $28.30 \%$ \\
Klebsiella pneumoniae & 8 & $15.09 \%$ \\
Enterobacter cloacae & 5 & $9.43 \%$ \\
Stenotrophomonas maltophilia & 4 & $7.55 \%$ \\
Citrobacter & 3 & $5.66 \%$ \\
Other Gram-negative bacteria & 10 & $18.87 \%$ \\
Fungi & 8 & $15.09 \%$ \\
Candida glabrata & 3 & $5.66 \%$ \\
Candida albicans & 3 & $5.66 \%$ \\
Candida tropicalis & 1 & $1.89 \%$ \\
Candida gaulli & 1 & $1.89 \%$ \\
\hline
\end{tabular}

Acinetobacter baumannii to antibacterial drugs was more than $65 \%$, and the resistance rate to piperacillin and minocycline was $100 \%$. The resistance rate of Klebsiella pneumonia to monoamidocyclines, carbapenems, aminoglycosamines and quinolones was less than $25 \%$, and the drug resistance rate to Cefoperazone/sulbactam was 0 (Table 3). The fungi were mainly Candida albicans and Candida glabrata, with high sensitivity to common antifungal agents (Table 4). All the patients in this study who had multi-drug resistant strains isolated did not accept a long-term application or extensive previous antimicrobial exposure.

\section{Risk Factors Analysis}

By using the univariate analysis, the results showed that the time of ECMO treatment, hospitalization, antibacterial drug use, ventilator use, catheter intubation and central venous intubation were significantly different in the study and the 
Table 3 Antimicrobial Resistance of Major Gram-Negative Bacteria

\begin{tabular}{|c|c|c|c|c|c|c|}
\hline \multirow[t]{2}{*}{ Antibacterials } & \multicolumn{3}{|c|}{ Acinetobacter baumannii $(n=15)$} & \multicolumn{3}{|c|}{ Klebsiella pneumoniae $(n=8)$} \\
\hline & Detected & Resistant & Resistance Rate (\%) & Detected & Resistant & Resistance Rate (\%) \\
\hline Piperacillin & 2 & 2 & 100.00 & 8 & 5 & 62.50 \\
\hline Piperacillin/tazobactam & 15 & 12 & 80.00 & 8 & I & 12.50 \\
\hline Ampicillin/sulbactam & 15 & 12 & 80.00 & 8 & 4 & 50.00 \\
\hline Cefoperazone/sulbactam & 6 & 5 & 83.33 & 3 & 0 & 0.00 \\
\hline Ceftazidime & 15 & 12 & 80.00 & 8 & I & 12.50 \\
\hline Cefepime & 15 & 12 & 80.00 & 8 & I & 12.50 \\
\hline Cefuroxime & - & - & - & 8 & 5 & 62.50 \\
\hline Cefazolin & - & - & - & 8 & 5 & 62.50 \\
\hline Cefatriaxone & 14 & II & 78.57 & 8 & 5 & 62.50 \\
\hline Cefotetan & - & - & - & 8 & I & 12.50 \\
\hline Aztreonam & - & - & - & 8 & I & 12.50 \\
\hline Meropenem & 15 & 12 & 80.00 & 8 & I & 12.50 \\
\hline Imipenem & 15 & 12 & 80.00 & 8 & I & 12.50 \\
\hline Minocycline & I & I & 100.00 & - & - & - \\
\hline Tobramycin & 14 & 11 & 78.57 & 8 & $\mathrm{I}$ & 12.50 \\
\hline Gentamicin & 15 & 12 & 80.00 & 8 & 2 & 25.00 \\
\hline Amikacin & 3 & 2 & 66.67 & 6 & I & 16.67 \\
\hline Ciprofloxacin & 12 & 9 & 75.00 & 8 & 2 & 25.00 \\
\hline Levofloxacin & 11 & 8 & 72.72 & 8 & I & 12.50 \\
\hline Sulfamethoxazole/trimethoprim & 14 & II & 78.57 & 8 & 4 & 50.00 \\
\hline
\end{tabular}

Note: - denotes no drug sensitivity test or natural drug resistance.

Table 4 Antimicrobial Resistance of Major Fungi

\begin{tabular}{|l|c|c|c|c|c|c|}
\hline \multirow{2}{*}{ Antimicrobial Agents } & \multicolumn{3}{|c|}{ Candida albicans } & \multicolumn{3}{c|}{ Candida glabrata } \\
\cline { 2 - 7 } & Detected & Resistant & Resistance Rate (\%) & Detected & Resistant & Resistance Rate (\%) \\
\hline Voriconazole & 2 & 0 & 0.00 & 3 & 1 & 33.3 \\
Fluconazole & 2 & 0 & 0.00 & 3 & 1 & 33.3 \\
\hline
\end{tabular}

control group $(p<0.05)$ (Table 5). The above influencing factors screened out by univariate analysis were subjected to multivariate analysis, and it was found that ECMO treatment time was an independent risk factor (Table 6).

ROC curve analysis represented that the AUC value of ECMO treatment time to predict nosocomial infection after ECMO was 0.840 (95\% CI 0.720-0.959, $p<$ $0.0001)$; the sensitivity was 0.553 ; and the specificity was 0.938; the maximum Youden's index was 0.490. The corresponding cut-off value was 4.5 days, which means that the risk of related nosocomial infection was increased when ECMO treatment time exceeded 4.5 days (Figure 1).

\section{Discussion}

In recent years, as ECMO technology rapidly develops in China, its technology, theory and practice are maturing. At present, ECMO technology has been carried out in many general hospitals; however, the prevention measures for its complications are still lacking experience due to various restrictive factors. ${ }^{4}$ During the ECMO support treatment of patients with severe cardiopulmonary failure, bacterial infection can prolong the time of ECMO and mechanical ventilation, and then increase the complication incidence, thus affecting the prognosis and mortality of patients with ECMO support treatment. ${ }^{16}$

During the treatment of ECMO patients, timely local strategies should be made to prevent nosocomial infections. In our clinical practice, the decision of experts from multiple departments including infectious diseases, microbiology, infectious disease control, critical care, respiratory, pharmacy and others have been collected to make the strategies of medication, infection prevention and infection 
Table 5 Univariate Analysis of the Nosocomial Infection in the Patients After ECMO

\begin{tabular}{|c|c|c|c|c|c|c|c|}
\hline \multirow[t]{2}{*}{ General Information } & \multicolumn{3}{|c|}{ Non-Infection Group $(n=40)$} & \multicolumn{2}{|c|}{ Infection Group ( $n=16)$} & \multirow[t]{2}{*}{$\mathbf{X}^{2} / Z$} & \multirow[t]{2}{*}{$\mathbf{P}$} \\
\hline & & Cases & Constituent Ratio (\%) & Cases & Constituent Ratio (\%) & & \\
\hline \multirow[t]{2}{*}{ Gender } & Male & 25 & 62.50 & 8 & 50.00 & \multirow[t]{2}{*}{0.738} & \multirow[t]{2}{*}{0.390} \\
\hline & Female & 15 & 37.50 & 8 & 50.00 & & \\
\hline Age & \multicolumn{3}{|c|}{$47.43 \pm 15.09$} & \multicolumn{2}{|r|}{$51.13 \pm 13.18$} & -0.86 & 0.40 \\
\hline \multirow[t]{2}{*}{ Hypertension } & No & 26 & 65.00 & 10 & 62.50 & \multirow[t]{2}{*}{0.031} & \multirow[t]{2}{*}{0.850} \\
\hline & Yes & 14 & 35.00 & 6 & 37.50 & & \\
\hline \multirow[t]{2}{*}{ Diabetes } & No & 34 & $85.00 \%$ & 12 & $75.00 \%$ & \multirow[t]{2}{*}{0.779} & \multirow[t]{2}{*}{0.377} \\
\hline & Yes & 6 & $15.00 \%$ & 4 & $25.00 \%$ & & \\
\hline \multirow[t]{2}{*}{ Treatment mode } & VA & 37 & $92.50 \%$ & 15 & $93.75 \%$ & \multirow[t]{2}{*}{0.027} & \multirow[t]{2}{*}{0.870} \\
\hline & VV & 3 & $7.50 \%$ & I & $6.25 \%$ & & \\
\hline \multirow[t]{2}{*}{ Installation } & Incision & 17 & $42.50 \%$ & 10 & $62.50 \%$ & \multirow[t]{2}{*}{$\mathrm{I} .83 \mathrm{I}$} & \multirow[t]{2}{*}{0.176} \\
\hline & Puncture & 23 & $57.50 \%$ & 6 & $37.50 \%$ & & \\
\hline \multirow[t]{2}{*}{ Discharge mode } & Normal & 34 & 85.00 & 15 & 93.75 & & \\
\hline & Death & 6 & 15.00 & I & 6.25 & & \\
\hline \multicolumn{2}{|l|}{ ECMO treatment time ${ }^{\#}$} & \multicolumn{2}{|r|}{$4.00(3.00 \sim 6.00)$} & \multicolumn{2}{|r|}{$9.50(5.25 \sim 12.50))$} & $-3.94 *$ & $<0.001$ \\
\hline \multicolumn{2}{|l|}{ Hospitalization time ${ }^{\#}$} & \multicolumn{2}{|r|}{$15.50(7.00 \sim 23.00)$} & \multicolumn{2}{|r|}{$55.50(21.25 \sim 83.75)$} & $-3.52 *$ & $<0.001$ \\
\hline \multicolumn{2}{|c|}{ Antibacterial drug use time\# } & \multicolumn{2}{|r|}{$8.00(5.00 \sim 17.00)$} & \multicolumn{2}{|r|}{$30.50(13.25 \sim 55.25)$} & $-3.87 *$ & $<0.001$ \\
\hline \multicolumn{2}{|l|}{ Ventilator use time $\mathrm{e}^{\#}$} & \multicolumn{2}{|r|}{$3.00(1.25 \sim 6.00)$} & \multicolumn{2}{|r|}{$11.00(3.00 \sim 24.50)$} & $-2.79 *$ & 0.005 \\
\hline Catheter intubation time & & & $7.00(4.00 \sim 11.00))$ & & $1.50(11.00 \sim 49.00)$ & $-4.26 *$ & $<0.001$ \\
\hline Central venous intubatic & ime & & $6.58 \pm 5.0 \mathrm{I}$ & & $25.00 \pm 17.70$ & $-6.08 *$ & $<0.001$ \\
\hline
\end{tabular}

Note: *Denotes $p<0.05$; ${ }^{\#}$ Denotes non-normal distribution after SK normality test.

Table 6 Multivariate Analysis of the Nosocomial Infection in the Patients After ECMO

\begin{tabular}{|l|c|c|c|c|c|c|c|}
\hline Related Factors & B & SE & $\begin{array}{l}\text { Wald } \\
\text { Value }\end{array}$ & OR Value & \multicolumn{3}{|c|}{$95 \%$ CI } \\
\hline Length of stay in the same period & -0.01 & 0.02 & 0.34 & 0.99 & 0.96 & 1.02 & 0.56 \\
Days of ECMO treatment & 0.36 & 0.17 & 4.38 & 1.44 & 1.02 & 2.02 & 0.04 \\
Days of antibiotic use & 0.08 & 0.09 & 0.74 & 1.08 & 0.91 & 1.28 & 0.39 \\
Days of ventilator use & 0.06 & 0.11 & 0.30 & 1.06 & 0.85 & 1.33 & 0.59 \\
Days of central venous intubation & 0.11 & 0.14 & 0.64 & 1.11 & 0.86 & 1.45 & 0.42 \\
Days of catheter intubation & -0.03 & 0.11 & 0.06 & 0.98 & 0.79 & 1.20 & 0.81 \\
\hline
\end{tabular}

Abbreviations: B, regression coefficient; SE, standard error; OR, odds ratio; Cl, confidence intervals; ECMO, extracorporeal membrane oxygenation.

control. Aditya et $\mathrm{al}^{12}$ evaluated the impact of an initial ECMO antimicrobial prophylaxis protocol (antimicrobials with a broader spectrum of activity) on antimicrobial use and NHSN reportable infection rates, the results showed that this multidisciplinary team-based approach to antimicrobial stewardship can significantly reduce antimicrobial prophylaxis and overuse in ECMO patients without increased risk of nosocomial infection. Therefore, it is extremely important to understand the related nosocomial infection after ECMO to prevent nosocomial infection occurrence. In the present study, 16 cases developed the nosocomial infection after ECMO, with an infection rate of $28.57 \%$, which is similar to the previous studies reporting that the infection incidence during ECMO is $20.5 \%$ $35.0 \%,{ }^{10,12,17}$ while our result is lower than the nosocomial infection rate of $40.62 \%$ evidenced by Wang et al. ${ }^{18}$

In this study, the main site of nosocomial infection after ECMO was located at the lower respiratory tract 
A

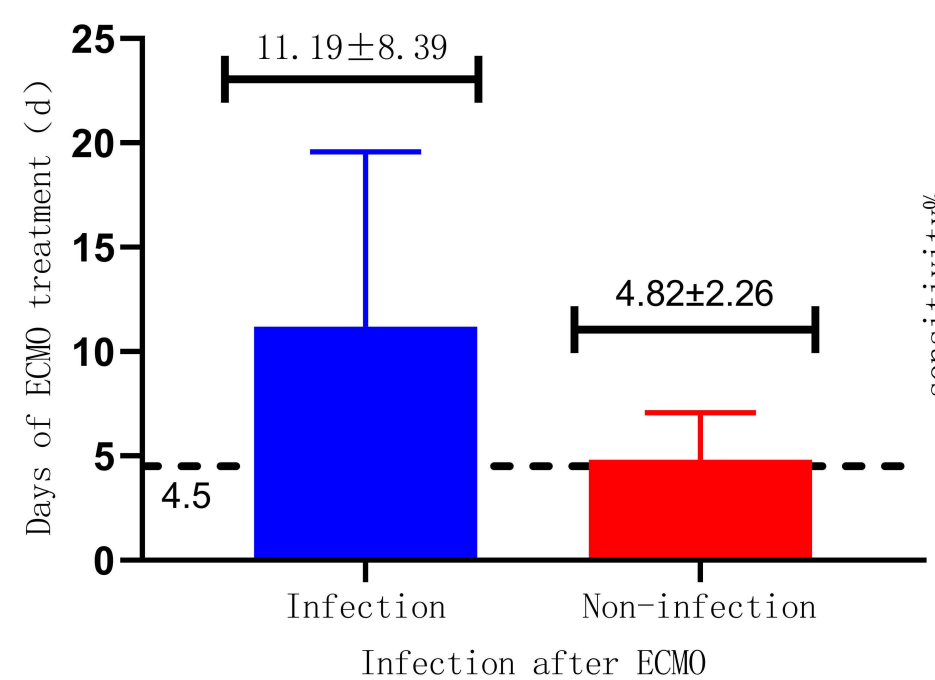

B

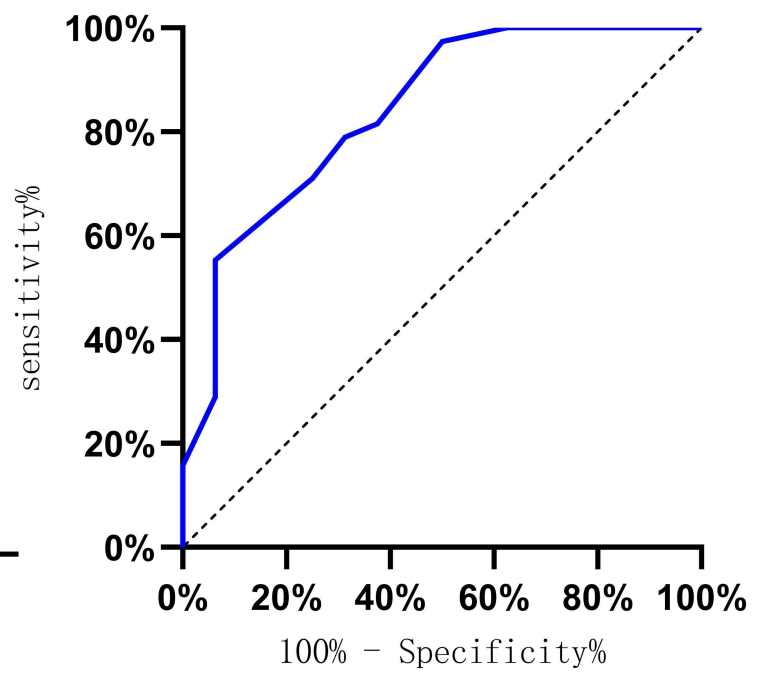

Figure I (A) Critical value of ECMO treatment time for predicting the risk of nosocomial infection after ECMO; (B) ROC curve analysis of the predictive value of ECMO treatment time on related nosocomial infections after ECMO.

infection, which was consistent with the results of previous studies. ${ }^{19-21}$ However, there is a study showing that the nosocomial infections of patients after ECMO support treatment are mainly bacteremia, followed by respiratory tract infection and urinary tract infection. ${ }^{22}$ This difference may be caused by individual characteristics, including population and preventive measures. We found that the rate of lower respiratory tract infection is much higher than in other parts. The use of the ventilator and retaining the tracheal cannula or incision may destroy the defense mechanism of the body; then, the gas can directly enter the lower respiratory tract, making the patient prone to lower respiratory tract infection. Therefore, it is necessary to timely assess the patient's condition and remove the cannula as soon as possible. Besides, no correlation was found between the age and infection risk in this study, which may be due to the fact that only adults were included in this study and the patients were mostly young.

In this study, 53 strains of pathogenic bacteria were detected in patients with nosocomial infection. Gramnegative bacteria were accounted for $84.91 \%$, and most of them were Acinetobacter baumannii and Klebsiella pneumonia. The above findings were in line with the results of a previous study, which indicated that Acinetobacter baumannii and Klebsiella pneumonia were the most common pathogenic bacteria. ${ }^{18}$ This study revealed that Acinetobacter baumannii mainly came from sputum samples, which was in accordance with a relevant domestic study, ${ }^{23}$ and it may be due to that sputum-contained nutrient can provide a good living environment for strains. Furthermore, the resistance rate of Acinetobacter baumannii to carbapenems was notably higher than the national average of 56.1\%. ${ }^{24}$ Amaya-Villar et $\mathrm{al}^{25}$ reported that polymyxins are the antimicrobials with the greatest level of in-vitro activity; Colistin is the antimicrobial most widely used; however, the side effect of renal toxicity needs to be considered. Moreover, Cefiderocol, ${ }^{26}$ a novel cephalosporin active against A. baumannii, may represent an attractive therapeutic option if ongoing clinical trials confirm preliminary results.

It has been reported that Klebsiella pneumonia shows high resistance to $\beta$-lactam antibiotics. ${ }^{27}$ Our results pointed that the resistance rate of Klebsiella pneumonia to carbapenems was higher than the national average of $10.1 \%{ }^{24}$ Klebsiella pneumonia is naturally resistant to ampicillin; in addition to that, Klebsiella pneumonia exhibits a high resistance rate to piperacillin, cefuroxime, cefazolin and ceftriaxone, ${ }^{28}$ but the resistance rate to Cefoperazone/sulbactam was the lowest. The province where our hospital located is the top five provinces of the national economy, and our hospital is the biggest first-class comprehensive hospital in this province, all the ECMO treatment was performed in the ICU department, which may lead to a relatively higher resistance rate level of Acinetobacter baumannii to carbapenems, compared to the national average. Timely understanding of pathogen distribution and drug resistance in patients with nosocomial infection, and understanding of the standardized treatment of Gram-negative bacilli infection can help to adjust the types and dosages of antibiotics according to the 
drug sensitivity results. The application of antibiotics, supplemented by active support treatment, can minimize and alleviate the generation of drug-resistant bacteria, which is conducive to disease treatment and control. However, Juthani et $\mathrm{al}^{29}$ considered that nosocomial infections have no effect on survival in adult ECMO patients, and the presence of either antibiotics or infection before ECMO does not affect developing nosocomial infections while on ECMO. Hence, further investigation is needed to fully elucidate the difference.

This study identified that ECMO treatment time was an independent risk factor. Moreover, the risk of infection will increase if ECMO treatment time exceeds 4.5 days. Similar results are reported by Tan et al. ${ }^{30}$ During the period of ECMO support treatment, invasive operations (such as intravenous intubation, tracheal intubation, and catheter intubation) are susceptible and high-risk factors. Patients with ECMO support are mostly in a high-stress state, which can easily lead to systemic inflammatory reactions and reduced immunity. With the extension of intubation days, bacteria breed and pathogen numbers increase, thus causing nosocomial infection. Patients with ECMO support treatment should be weaned in time when the condition permits. ${ }^{31}$ Wang et al ${ }^{32}$ demonstrated that nosocomial pneumonia during ECMO was associated with ventilator support duration before ECMO weaning, suggesting that medical history should be carefully considered.

\section{Conclusion}

In conclusion, according to the risk factors of nosocomial infection after ECMO, corresponding measures should be performed to control the occurrence of nosocomial infection. The aseptic operation should be strictly carried out, routine maintenance should be standardized, antibiotics should be reasonably used, and the patient's condition should be timely monitored. Patients receiving ECMO treatment for more than 4.5 days, have a higher risk of infection, who should be withdrawn from the machine and extubated if the condition permits to reduce the incidence of related nosocomial infection. In the follow-up study, multi-center will be combined to obtain sufficient samples and higher evidence results, and whether laboratory indicators have an impact on ECMO postoperative infection will be studied in the future.

\section{Ethical Statement}

The patient in our case has signed the informed consent. All patients provided informed consent. This study was designed following the Declaration of Helsinki and approved by the ethics committee of Jiangsu Shengze Hospital affiliated to Nanjing Medical University. The ethics number is 2019-SR-075

\section{Consent for Publication}

We had obtained from the patient for written informed consent for publication.

\section{Acknowledgments}

We would like to acknowledge the reviewers for their helpful comments on this paper. Zhan-Jie Li and DongFang Zhang are co-first authors for this study.

\section{Author Contributions}

All authors made substantial contributions to conception and design, acquisition of data, or analysis and interpretation of data; took part in drafting the article or revising it critically for important intellectual content; agreed to submit to the current journal; gave final approval for the version to be published; and agreed to be accountable for all aspects of the work.

\section{Funding}

This project was supported by the National Key R \& D Program Fund (2020YFC0848100), the Research Foundation for Infection Prevention and Control of Chinese Geriatrics Association (GRYJ-LRK2018016), and the Jiangsu Provincial Hospital Management Innovation Research Fund (JSYGY-3-2019-484).

\section{Disclosure}

The authors declare that they have no conflicts of interest.

\section{References}

1. Wang GN, Chen XF, Qiao L, et al. Comparison of extracorporeal and conventional cardiopulmonary resuscitation: a meta-analysis of 2260 patients with cardiac arrest. World J Emerg Med. 2017;8(1):5-11. doi:10.5847/wjem.j.1920-8642.2017.01.001.

2. Brodie D, Bacchetta M. Extracorporeal membrane oxygenation for ards in adults. $N$ Engl $J$ Med. 2011;365(20):14-1905. doi:10.1056/ NEJMct 1103720

3. Hill JD, O'Brien TG, Murray JJ, et al. Prolonged extracorporeal oxygenation for acute post-traumatic respiratory failure (shock-lung syndrome: use of the Bramson membrane lung). $N$ Engl J Med. 1972;286(12):629-634. doi:10.1056/NEJM197203232861204.

4. Zhang X, Zhuang J. Current situation and thinking of extracorporeal membrane oxygenation in China. Chin J Extracorporeal Circulation. 2017;15(02):68-71, 91.

5. Dongzi X, Yangzhaolian O. Analysis on standardization of extracorporeal membrane oxygenation at home and abroa. China Med Devices. 2020;35(6):150-153. 
6. Firstenberg MS, Stahel PF, Hanna J, et al. Successful Covid-19 rescue therapy by extra-corporeal membrane oxygenation (ECMO) for respiratory failure: a case Report. Patient Saf Surg. 2020;14 (4):20. doi:10.1186/s13037-020-00245-7.

7. Gao G, Hei F, Ji B, et al. Retrospective analysis of related complications in 128 adult patients with the support treatment of external membrane oxygenation. Mol Cardiol China. 2015;15(1):1197-1201.

8. Extracorporeal Life Support Organization (ELSO). ELSO ID task force recommendation summary. Ann Harbor, MI: ELSO; 2012: p. 8. Available from: https://www.elso.org/Portals/0/Files/ELSO-IDTask-Force-Recommendations-Summary.pdf. Accessed April 30, 2019.

9. Alberto Z, Giovanni L, Giuseppe B, et al. A meta-analysis of complications and mortality of extracorporeal membrane oxygenation. Crit Care Resusc. 2001;15(3):172-178.

10. Zhou H, Li C, Wang X, et al. Distribution and drug resistance of pathogens causing nosocomial infections in patients with extracorporeal membrane oxygenation treatment. Chin $J$ Nosocomiol. 2015;25(22):5097-5099.

11. Li B, Sun G, Cheng Z, et al. Analysis of nosocomial infections in post-cardiac surgery extracorporeal membrane oxygenation support therapy. Heart Surg Forum. 2018;21(5):E387-E391. doi:10.1532/ hsf.1789.

12. Aditya S, Priya S, Stevens RW, et al. Reducing broad spectrum antimicrobial use in Extracorporeal Membrane Oxygenation (ECMO): reduce AMMO Study. Clin Infect Dis. 2021:ciab118. doi:10.1093/cid/ciab118..

13. O'Horo JC, Cawcutt KA, De Moraes AG, Sampathkumar P, Schears GJ. The evidence base for prophylactic antibiotics in patients receiving extracorporeal membrane oxygenation. ASAIO J. 2016;62 (1):6-10. doi:10.1097/MAT.0000000000000287.

14. Hsu MS, Chiu KM, Huang YT, et al. Risk factors for nosocomial infection during extracorporeal membrane oxygenation. J Hosp Infect. 2009;73(3):210-216. doi:10.1016/j.jhin.2009.07.016.

15. (CLSI) Clinical And Laboratory Standards Institute. Performance Standards for Antimicrobial Susceptibility Testing. Twenty Seven Informational Supplement. 27th ed. M100S; 2019.

16. Bull T, Corley A, Smyth DJ, et al. Extracorporeal membrane oxygenation line-associated complications: in vitro testing of cyanoacrylate tissue adhesive and securement devices to prevent infection and dislodgement. Intensive Care Med Exp. 2018;6(1):6. doi:10.1186/ s40635-018-0171-8.

17. Zhou N, Bao M, Wang Z. Research progress of extracorporeal membrane oxygenation support therapy and prevention of complications. Clin Misdiagn Misther. 2018;31(4):106-111.

18. Wang J, Xiong Y, Shi Y, et al. Risk factors and etiology of nosocomial infection associated with extracorporeal membrane oxygenation in adult patients with cardiac problems after extracorporeal membrane oxygenation. Chin J Clin Infect Dis. 2019;38-43.

19. Sun G, Li B, Ye Z, et al. Risk factors of infection in patients receiving extracorporeal membrane oxygenation support therapy. Chin J Thorac Surg. 2016;3(4):209-215.
20. Zeng W, Xiao G, Zhong Z, et al. Risk factors of nosocomial infection after extracorporeal membrane oxygenation. Chin J Infect Control. 2014;13(4):212-214.

21. Xiaozu L, Binfei L, Geqin S, et al. Analysis of nosocomial infection after extracorporeal membrane oxygenation supportive treatment after cardiac surgery. South China J Cardiovasc Dis. 2018;24 (2):165-168.

22. Bizzarro MJ, Conrad SA, Kaufman DA, et al. Infections acquired during extracorporeal membrane oxygenation in neonates, children, and adults. Pediatr Crit Care Med. 2011;12(3):277. doi:10.1097/ PCC.0b013e3181e28894.

23. Wu G, Zhang G, Chen L, et al. Distribution and drug sensitivity of multi-drug-resistant bacteria in key departments of a hospital from 2016 to 2017. Clin J Med Officer. 2019;47(9):970-971, 974.

24. Committee of Expert on Rational Drug Use of National Health Commission of the P.R. China, Ministry of Health National Antimicrobial Resistance Investigation Net. China antimicrobial resistance surveillance system in 2018. China Licensed Pharmacist. 2020;017(1):1-10

25. Amaya-Villar R, Garnacho-Montero J. How should we treat Acinetobacter pneumonia? Curr Opin Crit Care. 2019;25 (5):465-472. doi:10.1097/MCC.0000000000000649.

26. El-Lababidi RM, Rizk JG. Cefiderocol: a Siderophore Cephalosporin. Ann Pharmacother. 2020;54(12):1215-1231. doi:10.1177/ 1060028020929988.

27. Zhu H, Yao J, Dong T, et al. Nosocomial infection and drug resistance of Klebsiella pneumoniae from 2011 to 2013. Chin J Health Lab Technol. 2015;586-589.

28. Gaiarsa S, Comandatore F, Gaibani P, et al. Genomic epidemiology of Klebsiella pneumoniae in Italy and novel insights into the origin and global evolution of its resistance to carbapenem antibiotics. Antimicrob Agents Chemother. 2015;59(1):389-396. doi:10.1128/ AAC.04224-14

29. Juthani BK, Macfarlan J, Wu J, Misselbeck TS. Incidence of nosocomial infections in adult patients undergoing extracorporeal membrane oxygenation. Heart Lung. 2018;47(6):626-630. doi:10.1016/j. hrtlng.2018.07.004.

30. Tan Y, Wang Y, Cai S, et al. Epidemiological characteristics of nosocomial infection in adult patients undergoing extracorporeal membrane oxygenation. Chin J Nosocomiol. 2020;30 (22):3434-3438.

31. Donker DW, Brodie D, Henriques JPS, Broomé M. Left ventricular unloading during veno-arterial ECMO: a review of percutaneous and surgical unloading interventions. Sage Choice. 2019;34(2). doi:10.1177/0267659118794112.

32. Wang $\mathrm{J}$, Huang $\mathrm{J}, \mathrm{Hu} \mathrm{W}$, et al. Risk factors and prognosis of nosocomial pneumonia in patients undergoing extracorporeal me mbrane oxygenation: a retrospective study. $J$ Int Med Res. 2020;48:300060520964701. doi:10.1177/0300060520964701.
Infection and Drug Resistance

\section{Publish your work in this journal}

Infection and Drug Resistance is an international, peer-reviewed openaccess journal that focuses on the optimal treatment of infection (bacterial, fungal and viral) and the development and institution of preventive strategies to minimize the development and spread of resistance. The journal is specifically concerned with the epidemiology of antibiotic resistance and the mechanisms of resistance development and diffusion in both hospitals and the community. The manuscript management system is completely online and includes a very quick and fair peerreview system, which is all easy to use. Visit http://www.dovepress.com/ testimonials.php to read real quotes from published authors. 